UDC 517.968.2

\author{
${ }^{1 *}$ Aisagaliev S., ${ }^{1}$ Zhunussova Zh., ${ }^{2}$ Akca H. \\ ${ }^{1}$ Al-Farabi Kazakh National University, Almaty, Kazakhstan \\ ${ }^{2}$ Abu-Dhabi University, Abu-Dhabi, UAE \\ *e-mail: Zhanat.Zhunusova@kaznu.kz
}

\title{
Solvability of a two-point boundary value problem with phase and integral constraints
}

\begin{abstract}
New mathematical techniques for considering the complex boundary value problems to solve topical problems of natural sciences, technology, economy and ecology etc. are needed. Mathematical models of nuclear and chemical reactors management processes, control of electric power and robotic systems, economic management and others are complex boundary value problems of ordinary differential equations. Boundary value problems are called complex if besides the boundary conditions there exist the phase constraints and integral constraints on the phase coordinates of the system. The main objectives are: the necessary and sufficient conditions for the existence of solutions of boundary value problems and the methods of construction of complex solutions of boundary value problems. The aim of the work is an attempt to create a unified theory of the study of solvability of boundary value problems and the construction of a general method for solving them, based on the use of modern computer technology. The work is devoted to solving the problems of boundary value problems of nonlinear systems of ordinary differential equations. We consider the boundary value problem with boundary conditions of the given convex closed sets. The necessary and sufficient conditions for existence of a solution of the problem and construction its solution are obtained. The basis of the proposed method for solving of the boundary value problem is a possibility to reduce to a class of linear Fredholm integral equation of the first kind [1]-[9]. Necessary and sufficient condition for existence of a solution of integral equation is proved. Fredholm integral equation of the first kind belongs to the insufficiently explored problems in mathematics [11][22].

Key words: integral equation, solvability, construction of a solution, extreme problem, functional gradient, minimizing sequence.
\end{abstract}

\section{Introduction}

We consider and find necessary and sufficient conditions for existence of a solution of the boundary value problem

$$
\begin{aligned}
& \dot{x}=A(t) x+\mu(t), t \in I=\left[t_{0}, t_{1}\right], \\
& \left(x\left(t_{0}\right)=x_{0}, x\left(t_{1}\right)=x_{1}\right) \in S \subset R^{2 n},
\end{aligned}
$$

at phase constraints

$$
\begin{gathered}
x(t) \in G(t) ; \\
G(t)=\left\{x \in R^{n} / \omega(t) \leq L(t) x \leq \phi(t),\right. \\
t \in I\},
\end{gathered}
$$

integral constraints

$$
\begin{gathered}
g_{j}(x) \leq c_{j}, \quad j=\overline{1, m_{1}}, \\
g_{j}(x)=c_{j}, \quad j=\overline{m_{1}+1, m_{2}},
\end{gathered}
$$

$$
g_{j}(x)=\int_{t_{0}}^{t_{1}}<a_{j}(t), x>d t, \quad j=\overline{1, m_{2}},
$$

here $A(t), L(t)$ are matrixes of $n \times n, s \times n$ order, accordingly, with piece-wise continuous elements, $S$ is the given closed set, $\omega(t), \varphi(t), t \in I$ are the prescribed continuous vector functions $s \times 1, a_{j}(t)$, $j=\overline{1, m_{2}}$ are the given piece-wise continuous vector functions of $n \times 1$ order, $c_{j}, j=\overline{1, m_{2}}$ are unknown constants, $t_{0}, t_{1}$ are the fixed time moments, $\mu(t)=\left(\mu_{1}(t), \ldots, \mu_{n}(t)\right)$ is the prescribed piece-wise continuous function, $\langle\cdot$,$\rangle is a scalar$ production. We construct a solution of the linear system (1) with boundary conditions (2).

We represent the matrix $A(t)$ of $n \times n$ order with piecewise continuous elements as the sum $A(t)=A_{1}(t)+B(t), t \in I$, that the matrix 


$$
W\left(t_{0}, t_{1}\right)=\int_{t_{0}}^{t_{1}} \Phi\left(t_{0}, t\right) B(t) B^{*}(t) \Phi^{*}\left(t_{0}, t\right) d t
$$

of $n \times n$ order be positive defined, where $\Phi(t, \tau)=\theta(t) \theta^{-1}(\tau), \theta(t)$ is a fundamental matrix solutions of the linear homogeneous system $\dot{\xi}=A_{1}(t) \xi$. We note, that the matrix $\theta(t)$ is a solution of the equation $\dot{\theta}(t)=A_{1}(t) \theta(t)$, $\theta\left(t_{0}\right)=I_{n}$, where $I_{n}$ is an unique matrix of $n \times n$ order. There are many options for representation the matrix $A(t)$ as the sum $A(t)=A_{1}(t)+B(t)$, $t \in I$ :

1. The matrix $A_{1}(t)$ can be chosen as a constant matrix $A_{1}$ of $n \times n$ order. In this case $\theta(t)=e^{A_{1} t}, t \in I$

2. The matrix $B(t)$ is chosen in the form $B(t)=B_{1}(t) P$, where $B_{1}(t)$ is the matrix of $n \times m$ order, $P$ is a constant matrix of $m \times n$ order, moreover $P=\left(I_{m}, 0_{m, n-m}\right)$, where $I_{m}$ is an unique matrix of $m \times m$ order, $0_{m, n-m}$ is a rectangular matrix of $m \times(n-m)$ order with zero elements.

Since the matrix $A(t)=A_{1}(t)+B(t), t \in I$, that equation (1) is written as

$$
\dot{x}=A_{1}(t) x+B(t) x+\mu(t), t \in I=\left[t_{0}, t_{1}\right] .
$$

In the case of a choice $B(t)=B_{1}(t) P$ the equation (3) has the form

$$
\dot{x}=A_{1}(t) x+B_{1}(t) P x+\mu(t), t \in I,
$$

where $B_{1}(t)$ is a matrix of $n \times m$ order, $P x$ is the vector function $m \times 1$. If $m=n$, then $P=I_{n}$, $B(t)=B_{1}(t), t \in I$. Without loss of generality, further we believe, that equation (4) is represented in the form (4), the matrix

$$
W_{1}\left(t_{0}, t_{1}\right)=\int_{t_{0}}^{t_{1}} \Phi\left(t_{0}, t\right) B_{1}(t) B_{1}^{*}(t) \Phi^{*}\left(t_{0}, t\right) d t
$$

In addition to (4), we consider the linear control system of the form

$$
\begin{gathered}
\dot{y}=A_{1}(t) y+B_{1}(t) u(t)+\mu(t), t \in I, \\
\left(y\left(t_{0}\right)=x_{0}, y\left(t_{1}\right)=x_{1}\right) \in S \subset R^{2 n}, \\
u(\cdot) \in L_{2}\left(I, R^{m}\right) .
\end{gathered}
$$

We note, that if $u(t)=\operatorname{Px}(t), t \in I$, then the system (6)-(8) coincides with the origin system (1), (2).

\section{Solution of a linear control system}

We use the following theorems 1 and 2. The theorems are proved in our previous works [1]-[9].

A solution of the boundary value problem relates to properties of the solutions of the following integral equation

$$
K u=\int_{t_{0}}^{t_{1}} K\left(t_{0}, t\right) u(t) d t=a, t \in I=\left[t_{0}, t_{1}\right],
$$

where $K\left(t_{0}, t\right)=\left\|K_{i j}\left(t_{0}, t\right)\right\|, i=\overline{1, n}, j=\overline{1, m}$ is the known matrix of $n \times m$ order with piecewise continuous by $t$ elements at fixed $t_{0}, t_{1}$, $u(\cdot) \in L_{2}\left(I, R^{m}\right)$ is the origin function, operator $K: L_{2}\left(I, R^{m}\right) \rightarrow R^{n}, a \in R^{n}$ is prescribed vector.

Theorem 1. Integral equation (2.1) at any fixed $a \in R^{n}$ has a solution if and only if the matrix

$$
C\left(t_{0}, t_{1}\right)=\int_{t_{0}}^{t_{1}} K\left(t_{0}, t\right) K^{*}\left(t_{0}, t\right) d t
$$

of $n \times n$ order is positive defined, where (*) is a transposition sign, $t_{1}>t_{0}$.

Theorem 2. Let $C\left(t_{0}, t_{1}\right)>0$ be a matrix. Then the general solution of integral equation (2.1) has the form

$$
\begin{gathered}
u(t)=K^{*}\left(t_{0}, t\right) C^{-1}\left(t_{0}, t\right) a+v(t)- \\
-K^{*}\left(t_{0}, t\right) C^{-1}\left(t_{0}, t_{1}\right) \int_{t_{0}}^{t_{1}} K\left(t_{0}, t\right) v(t) d t^{\prime}
\end{gathered}
$$

where $t \in I, \quad v(\cdot) \in L_{2}\left(I, R^{m}\right)$ is an arbitrary function, $a \in R^{n}$ is any vector. 
Theorem 3. Let the matrix $W_{1}\left(t_{0}, t_{1}\right)$ of $n \times n$ order be positive defined. Then control $u(\cdot) \in L_{2}\left(I, R^{m}\right)$ transfers the trajectory of system (6) from any initial point $y\left(t_{0}\right)=x_{0} \in R^{n}$ to any finite state $y\left(t_{1}\right)=x_{1} \in R^{n}$ if and only if

$$
\begin{gathered}
u(t) \in U=\left\{u(\cdot) \in L_{2}\left(I, R^{m}\right) / u(t)=\right. \\
v(t)+\lambda_{1}\left(t, x_{0}, x_{1}\right)+ \\
\left.+N_{1}(t) z\left(t_{1}, v\right), t \in I, \forall v, v(\cdot) \in L_{2}\left(I, R^{m}\right)\right\},
\end{gathered}
$$

where

$$
\begin{array}{cc}
\lambda_{1}\left(t, x_{0}, x_{1}\right)=B_{1}^{*}(t) \Phi^{*}\left(t_{0}, t\right) W_{1}^{-1}\left(t_{0}, t_{1}\right) a, & y(t)=z(t, v)+\lambda_{2}\left(t, x_{0}, x_{1}\right)+ \\
a=\Phi\left(t_{0}, t_{1}\right) x_{1}-x_{0}-\int_{t_{0}}^{t_{1}} \Phi\left(t_{0}, t\right) \mu(t) d t, & \text { where } t \in I, \\
\left.\lambda_{2}\left(t, x_{0}, x_{1}\right)=\Phi\left(t, t_{0}\right) W_{1}\left(t, t_{1}\right) W_{1}^{-1}\left(t_{0}, t_{1}\right) x_{0}+v\right) & +\Phi\left(t, t_{0}\right) W_{1}\left(t_{0}, t\right) W_{1}^{-1}\left(t_{0}, t_{1}\right) \Phi\left(t_{0}, t_{1}\right) x_{1}+ \\
+\int_{t_{0}}^{t} \Phi(t, \tau) \mu(\tau) d \tau-\Phi\left(t, t_{0}\right) W_{1}\left(t_{0}, t\right) W_{1}^{-1}\left(t_{0}, t_{1}\right) \int_{t_{0}}^{t_{1}} \Phi\left(t_{0}, t\right) \mu(t) d t
\end{array}
$$

$$
\begin{gathered}
N_{2}(t)=-\Phi\left(t, t_{0}\right) W_{1}\left(t_{0}, t\right) W_{1}^{-1}\left(t_{0}, t_{1}\right) \Phi\left(t_{0}, t_{1}\right), \\
W\left(t_{0}, t\right)=\int_{t_{0}}^{t} \Phi\left(t_{0}, \tau\right) B_{1}(\tau) B_{1}^{*}(\tau) \Phi^{*}\left(t_{0}, \tau\right) d \tau \\
W\left(t, t_{1}\right)=W\left(t_{0}, t_{1}\right)-W\left(t_{0}, t\right) .
\end{gathered}
$$

Proof. Solution of the system (6) has the form

$$
\begin{gathered}
y(t)=\Phi\left(t, t_{0}\right) x_{0}+\int_{t_{0}}^{t} \Phi(t, \tau) B_{1}(\tau) u(\tau) d \tau+ \\
+\int_{t_{0}}^{t} \Phi(t, \tau) \mu(\tau) d \tau, \quad t \in I
\end{gathered}
$$

Then the control which transfers the trajectory of system (6) from initial state $x_{0} \in R^{n}$ to the state $x_{1} \in R^{n}$ is defined by condition

$$
\begin{gathered}
y\left(t_{1}\right)=x_{1}=\Phi\left(t_{1}, t_{0}\right) x_{0}+ \\
+\int_{t_{0}}^{t_{1}} \Phi\left(t_{1}, t\right) B_{1}(t) u(t) d t+\int_{t_{0}}^{t_{1}} \Phi\left(t_{1}, t\right) \mu(t) d t
\end{gathered}
$$

$$
N_{1}(t)=-B_{1}^{*}(t) \Phi^{*}\left(t_{0}, t\right) W_{1}^{-1}\left(t_{0}, t_{1}\right) \Phi\left(t_{0}, t_{1}\right) .
$$

Function $z(t, v), \quad t \in I$ is a solution of the differential equation

$$
\begin{gathered}
\dot{z}=A_{1}(t) z+B_{1}(t) v(t), \quad z\left(t_{0}\right)=0, \\
v(\cdot) \in L_{2}\left(I, R^{m}\right) .
\end{gathered}
$$

Solution of differential equation (6) corresponding to control $u(t) \in U$ is defined by formula

This implies

$$
\begin{gathered}
\int_{t_{0}}^{t_{1}} \Phi\left(t_{1}, t\right) B_{1}(t) u(t) d t= \\
=x_{1}-\Phi\left(t_{1}, t_{0}\right) x_{0}-\int_{t_{0}}^{t_{1}} \Phi\left(t_{1}, t\right) \mu(t) d t
\end{gathered} .
$$

Since

$$
\Phi\left(t_{1}, t\right)=\Phi\left(t_{1}, t_{0}\right) \Phi\left(t_{0}, t\right),
$$

$\Phi^{-1}\left(t_{1}, t_{0}\right)=\Phi\left(t_{0}, t_{1}\right)$, that expression (12) is written in the form

$$
\begin{gathered}
\int_{t_{0}}^{t_{1}} \Phi\left(t_{0}, t\right) B_{1}(t) u(t) d t= \\
+\Phi\left(t_{0}, t_{1}\right) x_{1}-x_{0}-\int_{t_{0}}^{t_{1}} \Phi\left(t_{0}, t\right) \mu(t) d t=a
\end{gathered}
$$

Thus, the origin equation $u(\cdot) \in L_{2}\left(I, R^{m}\right)$ is a solution of the integral equation (13). Integral equation (13) can be represented as

$$
\begin{gathered}
K u=\int_{t_{0}}^{t_{1}} K\left(t_{0}, t\right) u(t) d t=a, \\
K\left(t_{0}, t\right)=\Phi\left(t_{0}, t\right) B_{1}(t), t \in I .
\end{gathered}
$$


As it follows from theorem 1, integral equation (13) has a solution if and only if the matrix

$$
\begin{gathered}
C\left(t_{0}, t_{1}\right)=\int_{t_{0}}^{t_{1}} K\left(t_{0}, t\right) K^{*}\left(t_{0}, t\right) d t= \\
=\int_{t_{0}}^{t_{1}} \Phi\left(t_{0}, t\right) B_{1}(t) B_{1}^{*}(t) \Phi^{*}\left(t_{0}, t\right) d t=W_{1}\left(t_{0}, t_{1}\right)
\end{gathered}
$$

of $n \times n$ order is positive defined. Consequently, the set $U \neq \varnothing, \varnothing$ is an empty set if and only if $W_{1}\left(t_{0}, t_{1}\right)>0$. It means, that the system (6)-(9) is controlled.
From theorem 2 it follows, that the general solution of the integral equation (13) has the form

$$
\begin{aligned}
& u(t)=K^{*}\left(t_{0}, t\right) C^{-1}\left(t_{0}, t_{1}\right) a+v(t)- \\
& -K^{*}\left(t_{0}, t\right) C^{-1}\left(t_{0}, t_{1}\right) \int_{t_{0}}^{t_{1}} K\left(t_{0}, t\right) v(t) d t
\end{aligned}
$$

where

$$
\begin{gathered}
K\left(t_{0}, t\right)=\Phi\left(t_{0}, t\right) B_{1}(t), \\
C\left(t_{0}, t_{1}\right)=W_{1}\left(t_{0}, t_{1}\right) .
\end{gathered}
$$

This implies

$$
u(t)=B_{1}^{*}(t) \Phi^{*}\left(t_{0}, t\right) W_{1}^{-1}\left(t_{0}, t_{1}\right) a+v(t)-B_{1}^{*}(t) \Phi^{*}\left(t_{0}, t\right) \times W^{-1}\left(t_{0}, t_{1}\right) \int_{t_{0}}^{t_{1}} \Phi\left(t_{0}, t\right) B_{1}(t) v(t) d t, t \in I
$$

where $v(\cdot) \in L_{2}\left(I, R^{m}\right)$ is any function. We note, that solution of differential equation (10) has the form

$$
\begin{gathered}
z(t)=z(t, v)=\Phi\left(t, t_{0}\right) z\left(t_{0}\right)+ \\
+\int_{t_{0}}^{t} \Phi(t, \tau) B_{1}(\tau) v(\tau) d \tau= \\
=\int_{t_{0}}^{t} \Phi(t, \tau) B_{1}(\tau) v(\tau) d \tau
\end{gathered}
$$

where $z\left(t_{0}\right)=0$. Consequently,

$$
\begin{gathered}
z\left(t_{1}\right)=z\left(t_{1}, v\right)=\int_{t_{0}}^{t_{1}} \Phi\left(t_{1}, t\right) B_{1}(t) v(t) d t= \\
=\Phi\left(t_{1}, t_{0}\right) \int_{t_{0}}^{t_{1}} \Phi\left(t_{0}, t\right) B_{1}(t) v(t) d t .
\end{gathered}
$$

From (14)-(16) it follows, that the origin control $u(t)=v(t)+\lambda_{1}\left(t, x_{0}, x_{1}\right)+N_{1}(t) z\left(t_{1}, v\right), t \in I$, $\forall v, v(\cdot) \in L_{2}\left(I, R^{m}\right)$.

This implies proposition of the theorem that $u(t) \in U$. Finally, inclusion (9) is proved.

Let $u(t) \in U$. Then solution of differential equation (6) has the form

$$
\begin{gathered}
y(t)=\Phi\left(t, t_{0}\right) x_{0}+\int_{t_{0}}^{t} \Phi(t, \tau) B_{1}(\tau)\left[v(\tau)+\lambda_{1}\left(\tau, x_{0}, x_{1}\right)+N_{1}(\tau) z\left(t_{1}, v\right)\right] d \tau+ \\
+\int_{t_{0}}^{t} \Phi(t, \tau) \mu(\tau) d \tau=\int_{t_{0}}^{t} \Phi(t, \tau) B_{1}(\tau) v(\tau) d \tau+\Phi\left(t, t_{0}\right) x_{0}+\int_{t_{0}}^{t} \Phi(t, \tau) B_{1}(\tau) \times \\
\times B_{1}^{*}(\tau) \Phi^{*}\left(t_{0}, \tau\right) d \tau W_{1}^{-1}\left(t_{0}, t_{1}\right)\left[\Phi\left(t_{0}, t_{1}\right) x_{1}-x_{0}-\int_{t_{0}}^{t_{1}} \Phi\left(t_{0}, t\right) \mu(t) d t\right]- \\
-\int_{t_{0}}^{t} \Phi(t, \tau) B_{1}(\tau) B_{1}^{*}(\tau) \Phi^{*}\left(t_{0}, \tau\right) d \tau W_{1}^{-1}\left(t_{0}, t_{1}\right) \Phi\left(t_{0}, t_{1}\right) z\left(t_{1}, v\right)=z(t, v)+ \\
+\lambda_{2}\left(t, x_{0}, x_{1}\right)+N_{2}(t) z\left(t_{1}, v\right), t \in I
\end{gathered}
$$

This implies representation of solution of the system (6) in the form (11). Theorem is proved.

It is easy to make sure in that

$$
\begin{aligned}
& y\left(t_{0}\right)=z\left(t_{0}, v\right)+\lambda_{2}\left(t_{0}, x_{0}, x_{1}\right)+N_{2}\left(t_{0}\right) z\left(t_{1}, v\right)=x_{0}, \\
& y\left(t_{1}\right)=z\left(t_{1}, v\right)+\lambda_{2}\left(t_{1}, x_{0}, x_{1}\right)+N_{2}\left(t_{1}\right) z\left(t_{1}, v\right)=x_{1} .
\end{aligned}
$$


Since proposition of the theorem is valid for any $x_{0} \in R^{n}, \quad x_{1} \in R^{n}$, that it is valid, when $\left(x_{0}, x_{1}\right) \in S \subset R^{2 n}$.

Lemma 1. Let $W_{1}\left(t_{0}, t_{1}\right)>0$ be a matrix. Then the boundary value problem (1), (2) is equivalent to the following problem:

$$
\begin{gathered}
v(t)+T_{1}(t) x_{0}+T_{2}(t) x_{1}+\bar{\mu}(t)+ \\
+N_{1}(t) z\left(t_{1}, v\right)=P y(t) \\
t \in I \\
\dot{z}=A_{1}(t) z+B_{1}(t) v(t), \quad z\left(t_{0}\right)=0, \\
t \in I \\
v(\cdot) \in L_{2}\left(I, R^{m}\right), \\
\left(x_{0}, x_{1}\right) \in S,
\end{gathered}
$$

where

$$
\begin{gathered}
T_{1}(t)=-B_{1}^{*}(t) \Phi^{*}\left(t_{0}, t\right) W_{1}^{-1}\left(t_{0}, t_{1}\right), \\
T_{2}(t)=B_{1}^{*}(t) \Phi^{*}\left(t_{0}, t\right) W^{-1}\left(t_{0}, t_{1}\right) \Phi\left(t_{0}, t_{1}\right), \\
\qquad I\left(v, x_{0}, x_{1}\right)=\int_{t_{0}}^{t_{1}} \mid v(t)+T_{1}(t) x_{0}+T_{2}(t) \\
\dot{z}=A_{1}(t) z+B_{1}(t) v(t), z\left(t_{0}\right)=0, t \in I, \\
v(\cdot) \in L_{2}\left(I, R^{m}\right),\left(x_{0}, x_{1}\right) \in S,
\end{gathered}
$$$$
I\left(v, x_{0}, x_{1}\right)=\int_{t_{0}}^{t_{1}}\left|v(t)+T_{1}(t) x_{0}+T_{2}(t) x_{1}+\bar{\mu}(t)+N_{1}(t) z\left(t_{1}, v\right)-P y(t)\right|^{2} d t \rightarrow \inf ,
$$

at conditions

where $y(t)=y\left(t, v, x_{0}, x_{1}\right), t \in I$ is defined by formula (23).

We note, that:

1. Functional $I\left(v, x_{0}, x_{1}\right) \geq 0$. Consequently, functional $I\left(v, x_{0}, x_{1}\right)$ is bounded below on the set $X=L_{2}\left(I, R^{m}\right) \times S$, where $\left(v, x_{0}, x_{1}\right) \in X \subset H$, $H=L_{2}\left(I, R^{m}\right) \times R^{2 n}$.

2. If $I\left(v_{*}, x_{0^{*}}, x_{1^{*}}\right)=0$, where $\left(v_{*}, x_{0^{*}}, x_{1^{*}}\right) \in X$, is a solution of the optimization problem (21)-(23), then

$$
\begin{gathered}
\bar{\mu}(t)=-B_{1}^{*}(t) \Phi^{*}\left(t_{0}, t\right) W_{1}^{-1}\left(t_{0}, t_{1}\right) \int_{t_{0}}^{t_{1}} \Phi\left(t_{0}, t\right) \mu(t) d t \\
\begin{array}{c}
y(t)=z(t, v)+C_{1}(t) x_{0}+C_{2}(t) x_{1}+ \\
+f(t)+N_{2}(t) z\left(t_{1}, v\right)
\end{array} \\
C_{1}(t)=\Phi\left(t, t_{0}\right) W_{1}\left(t, t_{1}\right) W_{1}^{-1}\left(t_{0}, t_{1}\right), \\
C_{2}(t)=\Phi\left(t, t_{0}\right) W_{1}\left(t_{0}, t\right) W_{1}^{-1}\left(t_{0}, t_{1}\right) \Phi\left(t_{0}, t_{1}\right), \\
f(t)=\int_{t_{0}}^{t} \Phi(t, \tau) \mu(\tau) d \tau- \\
-\Phi\left(t, t_{0}\right) W_{1}\left(t_{0}, t\right) W_{1}^{-1}\left(t_{0}, t_{1}\right) \int_{t_{0}}^{t_{1}} \Phi\left(t_{0}, t\right) \mu(t) d t .
\end{gathered}
$$

Proof of the lemma follows from theorem 3 and the equality $y(t)=x(t), \quad t \in I$, at $u(t) \in U$, $u(t)=P y(t), t \in I$. It is easy to make sure that expressions (17) - (19) are equivalent to the expressions (1), (2), at $W_{1}\left(t_{0}, t_{1}\right)>0$.

We consider the optimal control problem: minimize the functional 
$I\left(v_{*}, x_{0^{*}}, x_{1^{*}}\right)=0 . \quad$ Let $x\left(t ; t_{0}, x_{0^{*}}, x_{1^{*}}\right), \quad t \in I$, $\left(x_{0^{*}}, x_{1^{*}}\right) \in S$ be a solution of differential equation (1). As it follows from lemma 1 , boundary value problem (1), (2) is equivalent to the problem (17) (19). Hence,

$$
\begin{gathered}
v_{*}(t)+T_{1}(t) x_{0^{*}}+T_{2}(t) x_{1^{*}}+ \\
+\bar{\mu}(t)+N_{1}(t) z\left(t_{1}, v_{*}\right)=P y_{*}(t), \\
t \in I,
\end{gathered}
$$

$$
\begin{aligned}
\dot{z}\left(t, v_{*}\right)=A_{1}(t) z\left(t, v_{*}\right)+B_{1}(t) v_{*}(t), z\left(t_{0}\right)=0, \quad \text { Then } \\
I\left(v_{*}, x_{0^{*}}, x_{1^{*}}\right)=\int_{t_{0}}^{t_{1}}\left|v_{*}(t)+T_{1}(t) x_{0^{*}}+T_{2}(t) x_{1^{*}}+\bar{\mu}(t)+N_{1}(t) z\left(t_{1}, v_{*}\right)-P y_{*}(t)\right|^{2} d t=0,
\end{aligned}
$$

by identities (24), (25). Necessity is proved.

Sufficiency. Let $I\left(v_{*}, x_{0^{*}}, x_{1^{*}}\right)=0$ be the value.

We show, that boundary value problem (1), (2) has a solution. In fact, the value $I\left(v_{*}, x_{0^{*}}, x_{1^{*}}\right)=0$ if and only if the equality

$$
\begin{gathered}
v_{*}(t)+\lambda_{1}\left(t, x_{0^{*}}, x_{1^{*}}\right)+N_{1}(t) z\left(t_{1}, v_{*}\right)= \\
=P y\left(t, v_{*}, x_{0^{*}}, x_{1^{*}}\right)
\end{gathered}
$$

is held, where

$$
\begin{gathered}
y\left(t, v_{*}, x_{0^{*}}, x_{1^{*}}\right)=z\left(t, v_{*}\right)+\lambda_{2}\left(t, x_{0^{*}}, x_{1^{*}}\right)+ \\
+N_{2}(t) z\left(t_{1}, v_{*}\right), \\
t \in I .
\end{gathered}
$$

Function $y\left(t, v_{*}, x_{0^{*}}, x_{1^{*}}\right), \quad t \in I$ is solution of differential equation (6), at conditions (7), (8). Consequently,

$$
\begin{gathered}
t \in I, v_{*}(\cdot) \in L_{2}\left(I, R^{m}\right), \\
y_{*}(t)=z\left(t, v_{*}\right)+C_{1}(t) x_{0^{*}}+C_{2}(t) x_{1^{*}}+ \\
+f(t)+N_{2}(t) z\left(t_{1}, v_{*}\right) \\
t \in I
\end{gathered},
$$
$t \in I, y_{*}(t)=x\left(t ; t_{0}, x_{0^{*}}, x_{1^{*}}\right), t \in I$. is proved. Theorem is proved. function

where $\left(x_{0^{*}}, x_{1^{*}}\right) \in S, \quad u(t) \in U, \quad u(t)=P y_{*}(t)$,

$$
\begin{gathered}
\dot{y}\left(t, v_{*}, x_{0^{*}}, x_{1^{*}}\right)=A_{1}(t) y\left(t, v_{*}, x_{0^{*}}, x_{1^{*}}\right)+ \\
+B_{1}(t) u_{*}(t)+\mu(t)=A_{1}(t) y\left(t, v_{*}, x_{0^{*}}, x_{1^{*}}\right)+, \\
+B_{1}(t) P y\left(t, v_{*}, x_{0^{*}}, x_{1^{*}}\right)+\mu(t)
\end{gathered}
$$

where $u_{*}(t)=v_{*}(t)+\lambda_{1}\left(t, x_{0^{*}}, x_{1^{*}}\right)+N_{1}(t) z\left(t_{1}, v_{*}\right)$, $y\left(t_{0}\right)=x_{0^{*}}, \quad y\left(t_{1}\right)=x_{1^{*}}, \quad\left(x_{0^{*}}, x_{1^{*}}\right) \in S$, $u_{*}(t) \in U$. This implies, that $y\left(t, v_{*}, x_{0^{*}}, x_{1^{*}}\right)=x\left(t ; t_{0}, x_{0^{*}}, x_{1^{*}}\right), t \in I$ is solution of the boundary value problem (1), (2). Sufficiency

As it follows from theorem 4, if the value $I\left(v_{*}, x_{0^{*}}, x_{1^{*}}\right)>0$, then the boundary value problem (1), (2) has not solution. Thus, for constructing of a solution of boundary value problem (1), (2) necessary to solve optimization problem (21) - (23).

Lemma 2. Let $W_{1}\left(t_{0}, t_{1}\right)>0$ be a matrix,

$$
F_{0}(q, t)=\left|v+T_{1}(t) x_{0}+T_{2}(t) x_{1}+\bar{\mu}(t)+N_{1}(t) z\left(t_{1}\right)-P y\left(t, v, x_{0}, x_{1}\right)\right|^{2},
$$

where

$$
y\left(t, v, x_{0}, x_{1}\right)=z+C_{1}(t) x_{0}+C_{2}(t) x_{1}+f(t)+N_{2}(t) z\left(t_{1}\right), q=\left(v, x_{0}, x_{1}, z, z\left(t_{1}\right)\right) \in R^{m} \times R^{n} \times R^{n} \times R^{n} \times R^{n} .
$$

Then the partial derivatives

$$
\frac{\partial F_{0}(q, t)}{\partial v}=\left[2 v+T_{1}(t) x_{0}+T_{2}(t) x_{1}+\bar{\mu}(t)+N_{1}(t) z\left(t_{1}\right)-P y\right]
$$




$$
\begin{gathered}
\frac{\partial F_{0}(q, t)}{\partial x_{0}}=\left[2 T_{1}^{*}(t)-2 C_{1}^{*}(t) P^{*}\right]\left[v+T_{1}(t) x_{0}+T_{2}(t) x_{1}+\bar{\mu}(t)+N_{1}(t) z\left(t_{1}\right)-P y\right], \\
\frac{\partial F_{0}(q, t)}{\partial x_{1}}=\left[2 T_{2}^{*}(t)-2 C_{2}^{*}(t) P^{*}\right]\left[v+T_{1}(t) x_{0}+T_{2}(t) x_{1}+\bar{\mu}(t)+N_{1}(t) z\left(t_{1}\right)-P y\right], \\
\frac{\partial F_{0}(q, t)}{\partial z}=-2 P^{*}(t)\left[v+T_{1}(t) x_{0}+T_{2}(t) x_{1}+\bar{\mu}(t)+N_{1}(t) z\left(t_{1}\right)-P y\right], \\
\frac{\partial F_{0}(q, t)}{\partial z\left(t_{1}\right)}=\left[2 N_{1}^{*}(t)-2 N_{2}^{*}(t) P^{*}\right]\left[v+T_{1}(t) x_{0}+T_{2}(t) x_{1}+\bar{\mu}(t)+N_{1}(t) z\left(t_{1}\right)-P y\right] .
\end{gathered}
$$

Formulas (27) - (31) can be obtained by directly differentiating the function $F_{0}(q, t)$ by variable $q$.

Lemma 3. Let $W_{1}\left(t_{0}, t_{1}\right)>0$ be a matrix, set $S$ (22), (23);

1) Functional (21) is convex, at conditions be convex. Then:

$$
\text { 3) } \frac{\partial F_{0}(q, t)}{\partial q}=\left(\frac{\partial F_{0}(q, t)}{\partial v}, \frac{\partial F_{0}(q, t)}{\partial x_{0}}, \frac{\partial F_{0}(q, t)}{\partial x_{1}}, \frac{\partial F_{0}(q, t)}{\partial z}, \frac{\partial F_{0}(q, t)}{\partial z\left(t_{1}\right)}\right)
$$

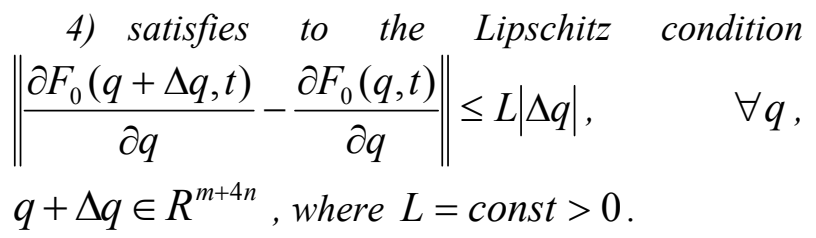

Proof. It is easy to make sure that

$$
\begin{gathered}
F_{0}(q, t)=q^{*} E^{*}(t) E(t) q+2 q^{*} E^{*}(t)[\bar{\mu}(t)- \\
-P f(t)]+[\bar{\mu}(t)-P f(t)]^{*}[\bar{\mu}(t)-P f(t)]
\end{gathered}, t \in I,
$$

where $E$ is a matrix of $m \times 4 n$ order. Then $\frac{\partial^{2} F_{0}(q, t)}{\partial q^{2}}=2 E^{*}(t) E(t) \geq 0$ for any $t, t \in I$.

Consequently, function $F_{0}(q, t)$ is convex with respect to $q$, i.e.

$$
\begin{gathered}
F_{0}\left(\alpha q^{1}+(1-\alpha) q^{2}, t\right) \leq \alpha F_{0}\left(q^{1}, t\right)+ \\
+(1-\alpha) F_{0}\left(q^{2}, t\right) \\
\forall q^{1}, q^{2} \in R^{m+4 n}, \forall \alpha, \alpha \in[0,1] .
\end{gathered}
$$

For any $v_{1}(\cdot) \in L_{2}\left(I, R^{m}\right), v_{2}(\cdot) \in L_{2}\left(I, R^{m}\right)$, and at all $\alpha>0, \alpha \in[0,1]$ the solution of differential equation (23) under $v_{\alpha}(t)=\alpha v_{1}(t)+(1-\alpha) v_{2}(t), \quad t \in I$ possesses by the property

$$
z\left(t, v_{\alpha}\right)=\alpha z\left(t, v_{1}\right)+(1-\alpha) z\left(t, v_{2}\right), t \in I .
$$

In fact,

$$
\begin{gathered}
z\left(t, v_{\alpha}\right)=\int_{t_{0}}^{t} \Phi(t, \tau) B_{1}(\tau) v_{\alpha}(\tau) d \tau=\int_{t_{0}}^{t} \Phi(t, \tau) B_{1}(\tau)\left[\alpha v_{1}(\tau)+(1-\alpha) v_{2}(\tau)\right] d \tau= \\
=\alpha \int_{t_{0}}^{t} \Phi(t, \tau) B_{1}(\tau) v_{1}(\tau) d \tau+(1-\alpha) \int_{t_{0}}^{t} \Phi(t, \tau) B_{1}(\tau) v_{2}(\tau) d \tau=\alpha z\left(t, v_{1}\right)+(1-\alpha) z\left(t, v_{2}\right), t \in I .
\end{gathered}
$$

Let $\xi_{1}=\left(v_{1}(t), x_{0}^{1}, x_{1}^{1}\right) \in X$, $\xi_{2}=\left(v_{2}(t), x_{0}^{2}, x_{1}^{2}\right) \in X$. Then the point

$$
\begin{gathered}
\xi_{\alpha}=\alpha \xi_{1}+(1-\alpha) \xi_{2}=\left(\alpha v_{1}+(1-\alpha) v_{2}, \alpha x_{0}^{1}+\right. \\
\left.+(1-\alpha) x_{0}^{2}, \alpha x_{1}^{1}+(1-\alpha) x_{1}^{2}\right) \in X
\end{gathered}
$$


The functional value

$$
\begin{gathered}
I\left(\xi_{\alpha}\right)=\int_{t_{0}}^{t_{1}} F_{0}\left(\alpha v_{1}+(1-\alpha) v_{2}, \alpha x_{0}^{1}+(1-\alpha) x_{0}^{2}, \alpha x_{1}^{1}+(1-\alpha) x_{1}^{2}, z\left(t, \alpha v_{1}+(1-\alpha) v_{2}\right),\right. \\
\left.z\left(t_{1}, \alpha v_{1}+(1-\alpha) v_{2}\right), t\right) d t=\int_{t_{0}}^{t_{1}} F_{0}\left(\alpha v_{1}+(1-\alpha) v_{2}, \alpha x_{0}^{1}+(1-\alpha) x_{0}^{2}, \alpha x_{1}^{1}+(1-\alpha) x_{2}^{2},\right. \\
\left.\alpha z\left(t, v_{1}\right)+(1-\alpha) z\left(t, v_{2}\right), \alpha z\left(t_{1}, v_{1}\right)+(1-\alpha) z\left(t_{1}, v_{2}\right), t\right) d t \leq \alpha \int_{t_{0}}^{t_{1}} F_{0}\left(\alpha q^{1}(t)+(1-\alpha) q^{2}(t)\right) d t \leq \\
\leq \alpha \int_{t_{0}}^{t_{1}} F_{0}\left(q^{1}(t), t\right) d t+(1-\alpha) \int_{t_{0}}^{t_{1}} F_{0}\left(q^{2}(t), t\right) d t=\alpha I\left(\xi_{1}\right)+(1-\alpha) I\left(\xi_{2}\right), \forall \xi_{1}, \xi_{2} \in X .
\end{gathered}
$$

In virtue by expressions (32), (33), where

$$
\begin{array}{r}
q^{1}(t)=\left(v_{1}(t), x_{0}^{1}, x_{1}^{1}, z\left(t, v_{1}\right), z\left(t_{1}, v_{1}\right)\right), \\
q^{2}(t)=\left(v_{2}(t), x_{0}^{2}, x_{1}^{2}, z\left(t, v_{2}\right), z\left(t_{1}, v_{2}\right)\right) .
\end{array}
$$

This implies the first proposition of the lemma. Since derivative

$$
\frac{\partial F_{0}(q, t)}{\partial q}=2 E^{*}(t) E(t) q+2 E^{*}(t)[\bar{\mu}(t)-P f(t)]
$$

that

$$
\frac{\partial F_{0}(q+\Delta q, t)}{\partial q}-\frac{\partial F_{0}(q, t)}{\partial q}=2 E^{*}(t) E(t) \Delta q,
$$

where $\Delta q=\left(\Delta v, \Delta x_{0}, \Delta x_{1}, \Delta z, \Delta z\left(t_{1}\right)\right), E^{*}(t) E(t)$ is the matrix of $(m+4 n) \times(m+4 n)$ order with piecewise continuous elements. Then

$$
\left\|\frac{\partial F_{0}(q+\Delta q, t)}{\partial q}-\frac{\partial F_{0}(q, t)}{\partial q}\right\| \leq L|\Delta q|,
$$

where $L=\sup _{t_{0} \leq t \leq t_{1}}\left\|E^{*}(t) E(t)\right\|>0$. Lemma is proved.

\section{Conclusion}

Scientific novelty of the results is that the origin problems are reduced to the corresponding Fredholm integral equations of the first kind. Necessary and sufficient conditions for existence of a solution of the integral equations are proved by theorem. It is shown, that the boundary problem of a linear system of ordinary differential equations can be reduced to the corresponding initial optimal control problems. From the solutions of initial value problems of optimal control can be obtained the following solutions: boundary value problems with constraints, boundary problems with a parameter, construction of periodic solutions of autonomous systems. The basis of the proposed methods for solving boundary value problems with different constraints is a possibility of reducing these problems to a class of linear Fredholm integral equation of the first kind. Fredholm integral equation of the first kind belongs to the insufficiently explored problems in mathematics. Therefore, fundamental research on integral equations and solution on their basis of boundary value problems of linear ordinary differential equations is a new promising direction in mathematics.

\section{References}

1. S.A. Aisagaliev. Controllability of a differential equation system. // Differential Equations. - 1991. Vol 27. - No. 9. - P. 1037-1045.

2. S.A. Aisagaliev., A.P. Belogurov. Controllability and speed of the process described by a parabolic equation with bounded control // Siberian Mathematical Journal. - 2012. - Vol. 53. No. 1. - P. 13-28.

3. S.A. Aisagaliev. Controllability theory of the dynamic systems. Almaty. Kazakh university. 2014. - P. 158.

4. S.A. Aisagaliev. Controllability and Optimal Control in Nonlinear Systems // Journal of Computer and Systems. - 1994. - No. 32. - P. 73-80. 
5. S.A. Aisagaliev., A.A. Kabidoldanova. Optimal control by dynamic systems // Palmarium Academic Publishing. - 2012. - Verlag. Germany. P. 288.

6. S.A. Aisagaliev., A.A. Kabidoldanova. On the Optimal Control of Linear Systems with Linear Performance Criterion and Constraints // Differential Equations. - 2012. - Vol. 48. - No. 6. P. 832-844.

7. S.A. Aisagaliev., T.S. Aisagaliev. Methods for solving the boundary value problems. Almaty. Kazakh university. 2002. - P. 348.

8. S.A. Aisagaliev., M.N. Kalimoldaev. Constructive method for solving a boundary value problem for ordinary differential equations // Differential Equations. - 2015. - Vol. 51. - Issue: 2. - P. 149-162.

9. S.A. Aisagaliev., Zh.Kh. Zhunussova. To the boundary value problem of ordinary differential equations // Electronic Journal of Qualitative Theory of Differential Equations. - 2015. - No. 57. - P. 1-17.

10. V.I. Smirnov. // Course of higher mathematics. M. Science. - 1974. - Vol. 4. - P. 336.

11. A.N. Kolmogorov., S.V. Fomin. Elements of the function theory and functional analysis // Science. - 1989. - P. 624.

12. M.L. Krasnov. Integral equations. İ: Science. $-1975 .-$ P.304.

13. A.N. Tikhonov., V.Ya. Arsenin. Methods for solving of the ill-posed problems. Ì: Science. 1986. - P. 288.
14. M.M. Lavrentev., About some ill-posed problems of mathematical physics // RAS. - 1962. P. 305.

15. V.K. Ivanov. On Fredholm integral equations of the first kind // Differential equations. 1967. - Vol. 3. - No. 3. - P. 21-32.

16. V.M. Fridman. Method of successive approximations for Fredholm integral equations of the first kind // UMN XI. - 1956. - Vol. 1. - P. 5685 .

17. F.M. Mors., G. Feshbah., Methods of mathematical physics. - 1958. - Vol. 2. - P. 536.

18. S.A. Aisagaliev. General solution of a class integral equations // Mathematical journal. Institute of Mathematics MES RK. - 2005. - Vol. 5. - No. 4. - P. 7-13.

19. S.A. Aisagaliev. Constructive theory of the optimal control boundary value problems. Almaty: Kazakh university. 2007. - P. 328.

20. S.A. Aisagaliev., A.P. Belogurov. I.V. Sevrugin. To solution of Fredholm integral equations of the first kind for function with several variables // Bulletin KazNU. - 2011. - No. 1. - P. 316.

21. S.A. Aisagaliev. Lectures on optimal control. Almaty: Kazakh University. 2007. - P. 278.

22. S.A. Aisagaliev., I.V. Sevrugin. Controllability and high-speed performance of processes described by ordinary differential equations // Bulletin KazNU. - 2014. - No. 2. P. 20-37. 